- ACORN AESTrallan COLLGe of | JOURNAL OF PERIOPERATIVE NURSING

\title{
The effectiveness of a telehealth program on the anxiety levels among women with core needle biopsy for suspected breast cancer
}

Follow this and additional works at: https://www.journal.acorn.org.au/jpn

Part of the Nursing Commons

(c) (1)

This work is licensed under a Creative Commons Attribution 4.0 License.

\section{Recommended Citation}

Ruttanamontree, Sansanee; Asdornwised, Usavadee; Wongkongkam, Kessiri; and Chuthapisith, Suebwong (2020) "The effectiveness of a telehealth program on the anxiety levels among women with core needle biopsy for suspected breast cancer," Journal of Perioperative Nursing: Vol. 33 : Iss. 2 , Article 4. Available at: https://doi.org/10.26550/2209-1092.1071

https://www.journal.acorn.org.au/jpn/vol33/iss2/4

This Article is brought to you for free and open access by Journal of Perioperative Nursing. It has been accepted for inclusion in Journal of Perioperative Nursing by an authorized editor of Journal of Perioperative Nursing. 


\section{Authors}

Ms Sansanee Ruttanamontree MSN candidate

Faculty of Nursing and Faculty of Graduate Studies, Mahidol University

Assoc. Prof. Dr Usavadee Asdornwised PhD

Faculty of Nursing, Mahidol University

Dr Kessiri Wongkongkam

PhD

Faculty of Nursing, Mahidol University Assoc. Prof. Dr Suebwong Chuthapisith PhD, MD

Head of Head, Neck and Breast Surgery Division, Surgical Department, Faculty of Medicine, Mahidol University

\section{Corresponding author}

Assoc. Prof. Dr. Usavadee Asdornwised Faculty of Nursing, Mahidol University 2 Wanglang Rd

Bangkoknoi, Bangkok, 10700

Thailand

Email: usavadee.asd@mahidol.ac.th

\section{The effectiveness of a telehealth program on the anxiety levels among women with core needle biopsy for suspected breast cancer}

\author{
Abstract \\ Aim \\ This study aimed to investigate the effectiveness of a telehealth program on \\ anxiety levels among women underging core needle biopsy for suspected \\ breast cancer.
}

Samples and setting

A quasi-experimental research study was conducted at a university hospital in Bangkok, Thailand. Seventy women with breast lumps scheduled for a core needle biopsy were enrolled.

\section{Methods}

Thirty-five women with breast lumps were assigned to participate in the telehealth program plus routine care, while another group of thirty-five women received only routine care. Data was collected using a demographic data questionnaire and visual analogue scale (VAS). These tools assessed the patients' anxiety levels once before participating in the program (on admission to hospital) and twice after participating in the program (before the biopsy and on the day of follow-up). The telehealth program consisted of educating, consulting and monitoring patients via the LINE messaging application and handbooks for women receiving a core needle biopsy with routine care.

\section{Results}

The study group had statistically lower anxiety levels than that of the control group both before biopsy $(p=0.003)$ and on the day of follow-up $(p=0.001)$. In addition, the study group had statistically lower anxiety levels after participating in the program, both before biopsy $(p=0.045)$ and on the day of follow-up $(p=0.002)$.

\section{Conclusions}

The telehealth program can be used to care for patients that have been scheduled for core needle biopsy in order to reduce their anxiety.

Keywords: anxiety, core needle biopsy, telehealth program 


\section{Introduction}

Breast cancer is frequently found in women worldwide and is the second leading cause of death ${ }^{1}$, with trends showing an expected rise to 3.1 per cent by $2025^{2}$. In Thailand, breast cancer incidence is found in 28.5 out of 100000 women $^{3}$. Breast cancer is considered the most common cancer in women. The diagnosis of a breast lump as cancer occurs at a rate of 27-34 per cent 4,5 . Diagnoses currently occurs using the triple assessment guideline, consisting of clinical follow-up, a breast imaging study (such as ultrasound or mamograms) and pathology follow-up (such as fine needle biopsy, core needle biopsy or excisional biopsy ${ }^{6}$ ). Breast cancer is the second most frequently encountered diagnosis among patients visiting the hospital taking part in this study, Mahidol University hospital. According to hospital statistics, during the years 2016 to 2018, almost 6000 suspected breast cancer patients were diagnosed using a core needle biopsy?

A core needle biopsy is an outpatient procedure for breast cancer diagnosis ${ }^{8}$. This procedure can take 30 minutes to two hours. Typically, a diagnosis will occur within ten days. Those patients with core needle biopsy receive short-term care in the hospital such as health counselling, advice and support. However, the transitional care between hospital and home has not been explicitly established for core needle biopsy patients. In addition, the transitional care for the outpatient surgery group still does not provide complete care. This may result in patients' experiencing complications or dissatisfaction with their service?

Forty-two to 50 per cent of women that have suspected breast cancer and require core needle biopsy were found to have anxiety ${ }^{10}$. This anxiety continues to occur until the patients receive the biopsy results ${ }^{11}$.
The factors influencing anxiety include the waiting time before receiving the core needle biopsy ${ }^{12}$, the experience of having suspected breast cancer or a biopsy, a family history of breast cancer and lack of knowledge about the disease, biopsy methods, the time required to receive the results, the risk of developing breast cancer and information about follow-up and diagnostic tests ${ }^{13}$. Increased anxiety may affect a woman's ability to decide whether to use health care or lead to them refusing treatment, searching for other treatment methods or refusing follow-up care. These decisions could possibly cause the disease to become more severe. Additionally, the women's anxiety can interfere with the function of their perception, memory and decision-making regarding treatment which may cause them to receive incorrect information and feel unable to make informed treatment decisions or perform selfcare after treatment ${ }^{14}$.

Literature reviewed for the study showed that care composed of health information and psychological support can reduce patients' anxiety during the breast cancer diagnostic period $^{15}$. Telehealth programs have become increasingly popular using devices such as computers, tablets or smartphones to communicate with patients for preoperative or long-term follow-up care ${ }^{16}$. Telehealth programs can also be used for surgical patients or as part of perioperative preparation ${ }^{17}$. Moreover, telehealth programs can be used for patients with psychological conditions by providing educational material about their treatment or consultation via online media ${ }^{18}$. In Thailand, telehealth programs have not been consistently used in the care of patients, meaning that patient care ends on leaving the hospital. According to a study on the effects of discharge planning for women that received breast mass excision, patients were not monitored until receiving the diagnosis ${ }^{19}$ and the program ended only one day after the surgery. Additionally, a study on the effects of providing preoperative information to women with breast cancer based on nursing practice guidelines for anxiety ended the program on the date of the surgery. Furthermore, patients were not monitored at home after the surgery ${ }^{20}$, which may have led to inconsistencies in their care.

The present study applied a transitional care model (TCM) providing consistent pre- and posthospital care for patients who were treated at the hospital ${ }^{21}$. This model is dependent on the cooperation of the health care teams in meeting the patients' needs. The TCM can be used as a conceptual framework in caring for women receiving core needle biopsy for suspected breast cancer. It can also help nurses in providing physical and psychological care for patients, assessing problems, evaluating needs and solving potential problems when patients are staying at home. In Thailand, this approach to care and monitoring for women in this population has been rare. Therefore, this study examines a telehealth program for reducing anxiety in women undergoing core needle biopsy for suspected breast cancer.

\section{Aim}

The aim of the study was to investigate the effectiveness of a telehealth program on the anxiety levels among women undergoing core needle biopsy for suspected breast cancer.

\section{Samples and setting}

The population in this study consisted of female patients aged 18 years and older with breast lumps who had a planned core needle biopsy with local anaesthetic. Inclusion criteria consisted of 
patients receiving a core needle biopsy for the first time. All of the participants had a waiting time of no more than four weeks to receive the core needle biopsy. They had the ability to perceive, understand and communicate in the Thai language and use a smartphone. Exclusion criteria consisted of patients already diagnosed with psychiatric disorders, such as anxiety disorder and depression, and cognitive disorders or patients diagnosed with other types of cancer.

The sample size was calculated using power analysis with the $\mathrm{G}^{*}$ Power program. The power of the test was $0.80, \alpha=0.05$. A review of similar studies $^{22}$ indicated an effect size of 0.67 . Hence, the sample size was calculated at 29 samples per group. In order to prevent sample loss, the researcher added an attrition rate of 20 per cent. Therefore, the number of participants was 35 in each group. This prospective research was conducted from January to June in 2019 at Mahidol University hospital in Bangkok.

\section{Methods}

This quasi-experimental research had a two-group, pretest-posttest design. The research intervention consisted of the telehealth program for patients receiving a core needle biopsy. The researcher and advisory professors developed this program. It was conceptualised using the TCM. The health care team consisted of the physician and nurses who were promoting health outcomes throughout the period from arriving at hospital to returning home ${ }^{21}$. The program consisted of education about the core needle biopsy, consulting with patients, and monitoring their symptoms after receiving the core needle biopsy by sending messages via the LINE application. In addition, the participants were provided with a handbook with

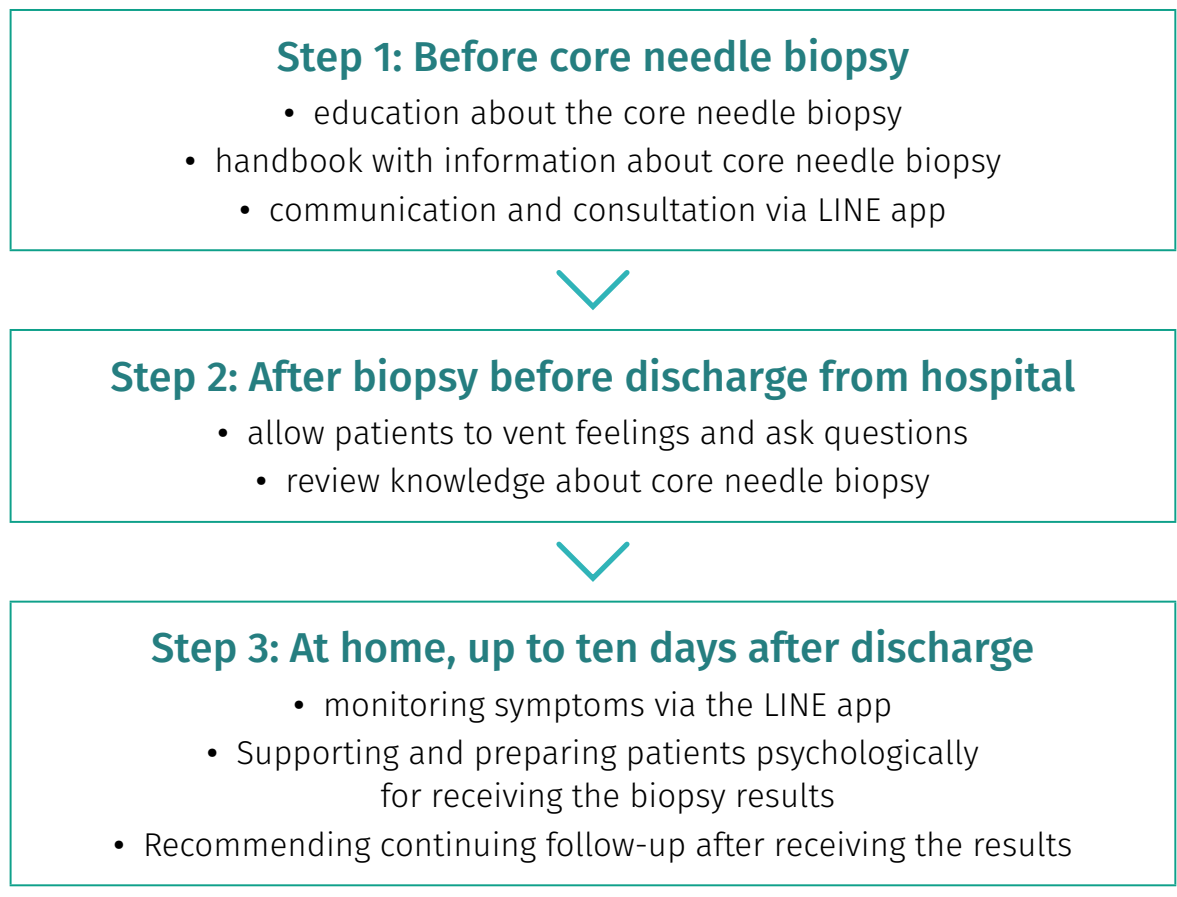

Figure 1: Flowchart of telehealth program for women receiving the core needle biopsy

content related to core needle biopsy methods, the necessary steps to be taken, instructions for before and after the core needle biopsy, and communication channels for asking questions (Figure 1). Three qualified experts tested the material for content validity. The telehealth program was tested with ten participants who were representative of the sample group in order to assess the understanding of the content before the program was used with the participants.

The data collection instruments consisted of a demographic data recording form consisting of age, religion, marital status, level of education, occupation, income, treatment rights and medical history data. The visual analogue scale (VAS) was used to assess the patients' anxiety levels with one question for the participants to assess their current anxiety ${ }^{23}$. The VAS is widely used and assesses anxiety in clinical practice settings. The scale was linear and horizontal with a length of $100 \mathrm{~mm}$, beginning from the left side at 'no anxiety' and rising toward the right to 'highest anxiety.'

\section{Data collection method}

After ethical approval from the Institutional Review Board at Mahidol University, the researcher selected the participants at the breast clinic based on the inclusion criteria. The researcher inquired about the subjects' willingness to participate in the research project along with an explanation of the research objectives and informed consent. The subjects then signed the forms consenting to participation.

The researcher collected data in two stages, firstly from the thirty-five participants in the control group and then the thirty-five participants in the study group, in order to prevent contamination between the two groups. The control group received routine care without access to the telehealth program. The study group received the same routine care plus access to the telehealth program consisting of education and 
Table 1: Comparison of study group and control group anxiety level mean scores with independent t-test statistics

\begin{tabular}{|c|c|c|c|c|c|c|c|c|c|c|}
\hline \multirow[b]{2}{*}{ Anxiety level } & \multicolumn{4}{|c|}{ Study group $(n=35)$} & \multicolumn{4}{|c|}{ Control group ( $n=35)$} & \multirow[b]{2}{*}{$\mathrm{T}$} & \multirow[b]{2}{*}{ p-value } \\
\hline & $\min$ & $\max$ & mean & S.D. & $\min$ & $\max$ & mean & S.D. & & \\
\hline At admission (TO) & 0.8 & 10 & 5.61 & 2.86 & 1.2 & 9.6 & 5.66 & 2.20 & 0.084 & 0.930 \\
\hline Before biopsy (T1) & 0.4 & 8.6 & 4.83 & 2.17 & 2.6 & 9.3 & 6.29 & 1.86 & 2.634 & $0.003^{*}$ \\
\hline Day of follow-up (T2) & 0 & 9 & 3.83 & 2.43 & 1.6 & 10 & 5.79 & 2.44 & 3.158 & $0.001^{*}$ \\
\hline
\end{tabular}

${ }^{*} p<.05$ S.D. $=$ standard deviation

a handbook about the core needle biopsy, consultation and monitoring of symptoms after receiving the core needle biopsy and when they had returned home via the LINE app.

The participants completed their own demographic and medical history data, while the researcher completed the data from the patient's medical records. The VAS was completed by the participants at three time points: on the day of admission, on the day of receiving the core needle biopsy and on the day of follow-up before receiving the biopsy result.

\section{Data analysis}

SPSS version 20 was used for data analysis. Differences in demographic data and treatment background data were compared using a chi-squared test, Fisher's exact test and an independent t-test. The mean scores for patient anxiety were compared between the study group and the control group by using independent t-test and mean levels of anxiety before and after the telehealth program, and were then compared with repeated measure ANOVA statistics.

\section{Results}

\section{Demographic and medical history data}

Concerning the participants' demographic data, the study group was found to have a mean age of 50.63 years $(S D=7.85)$ and the control group was found to have a mean age of 52.60 years $(S D=10.22$ ) Most participants had completed a bachelor degree or higher as their highest degree of education (57.2\% in the study group and $60.0 \%$ in the control group). Most participants worked as a government official or university officer ( $40 \%$ in the study group and $42.9 \%$ in the control group) and had mean family, monthly incomes amounting to 30000 baht or greater (60\% in the study group and $42.8 \%$ in the control group). The health care costs of about half the participants were provided by the government (51.5\% in both groups) and less than half the participants heard about this treatment from physicians and nurses (40\% in the study group and $48.6 \%$ in the control group).

Regarding the medical history in both groups, most participants were found to have no chronic diseases, no history of using birth control, no history of breast symptoms, no family history of cancer and had previously conducted breast self-examination. When the demographic and medical history data were compared between both groups, no differences were found, with statistical significance of 0.05 .

\section{Anxiety levels}

Table 1 shows the mean scores for anxiety between the group who participated in the telehealth program and the group who received routine care only. The study group was found to have a lower anxiety level $(4.83 \pm 2.17)$ than the control group $(6.29 \pm 1.86)$ before the core needle biopsy ( $p=0.003$ ), in addition to having a lower anxiety level than the control group on the day of follow-up (study group: $3.83 \pm 2.43$, control group: $5.79 \pm 2.44)$. This was statistically significant $(p=0.001)$.

Table 2 compares the mean anxiety scores for the two groups at admission (TO), before the core needle biopsy (T1) and on the day of follow-up (T2). The scores for the study group were found to be different to those for the control group, with a statistical significance for at least one pair $(F=11.085, p=$ 0.000).

Table 3 compares the mean anxiety scores for the study group before and after participating in the telehealth program. The study group was found to have statistically significant lower mean anxiety scores after participating in the telehealth program, both during the period before the core needle biopsy ( $p$ $=0.045)$ and on the day of followup ( $p=0.002)$, than at admission before participating in the telehealth program.

During the telehealth program, some of the participants shared thoughts via the LINE app about potential causes of anxiety, including being suspicious of breast cancer results, pain during and after the biopsy, and inability to take care of themselves after the biopsy. Two participants 
Table 2: Comparison of study group and control group anxiety level mean scores using repeated measure ANOVA

\begin{tabular}{|c|c|c|c|c|c|c|c|c|}
\hline \multirow[b]{2}{*}{ Anxiety level } & \multicolumn{2}{|c|}{ At admission (T0) } & \multicolumn{2}{|c|}{ Before biopsy (T1) } & \multicolumn{2}{|c|}{ Day of follow-up (T2) } & \multirow[b]{2}{*}{$\mathrm{F}^{a}$} & \multirow[b]{2}{*}{$\mathrm{p}$-value } \\
\hline & mean & S.D. & mean & S.D. & mean & S.D. & & \\
\hline Control group & 5.66 & 2.20 & 6.29 & 1.86 & 5.79 & 2.44 & 1.227 & .294 \\
\hline Study group & 5.61 & 2.86 & 4.83 & 2.17 & 3.83 & 2.43 & 11.085 & $.000^{*}$ \\
\hline
\end{tabular}

${ }^{a}$ Greenhouse-Geisser correction, ${ }^{*} p<.05$

sent images to ask questions about wound healing.

\section{Discussion}

The women who received a core needle biopsy and had access to the telehealth program had lower anxiety levels than the women receiving only routine care; the difference was statistically significant $(p<0.05)$. The participants in the study group had lower mean anxiety scores than those in the control group before the core needle biopsy (T1) and on the day of follow-up (T2) because the telehealth program allowed them to manage their emotional responses, fear and anxiety. In addition, the program supported and provided opportunities for the participants to ask questions and relieve anxieties. Moreover, the telehealth program provided a degree of physiological and psychological readiness for the patients preparing for core needle biopsy. The continuing care of patients based on the TCM was conceptualised in this study. The gap in nursing care was covered by using the telehealth program, which decreased anxiety levels in participants as a result.

Regarding the anxiety characteristics among the participants that received the core needle biopsy in this study, the patients were noted to have anxiety at every stage. Upon admission, the patients in the control group were found to have no differences in their mean anxiety scores from the study group. Furthermore, the mean anxiety scores were found to have increased before the core needle biopsy and decreased on the day of follow-up. Additionally, the telehealth program included transitional care, which consisted of anxiety assessments, education, consultation and consistent monitoring of participants via the LINE application after they went home. The program helped the participants receive continuing care, which decreased their anxiety levels.

Table 3: Comparison of anxiety level mean scores in the study group at admission (T0), before core needle biopsy (T1) and on the day of followup (T2) in matched pairs categorised by time period using multiple comparison statistics by the Bonferroni method $(\mathrm{N}=35)$

\begin{tabular}{|l|l|l|l|l|} 
Pair comparison for & & \multicolumn{2}{|l|}{$95 \%$ confidence interval } \\
\cline { 4 - 5 } $\begin{array}{l}\text { anxiety level mean } \\
\text { scores by time period }\end{array}$ & $\begin{array}{l}\text { mean } \\
\text { difference }\end{array}$ & p-value & lower & upper \\
\hline T0 and T1 & 0.777 & $0.045^{\star}$ & 0.013 & 1.541 \\
\hline T0 and T2 & 1.774 & $0.002^{\star}$ & 0.574 & 2.974 \\
\hline T1 and T2 & 0.997 & $0.014^{*}$ & 0.165 & 1.829 \\
\hline
\end{tabular}

${ }^{*} p<0.05$

According to Dermia et al. ${ }^{9}$ who studied the experiences of patients who had undergone breast biopsy, most patients felt anxiety because of a lack of knowledge and information related to the disease and its procedure. The patients also expressed fear and concern which was related to spiritual needs. In addition, Brocken et al..$^{24}$ and Harding ${ }^{25}$ studied the suffering and related factors during the diagnostic phase of suspected cancer. They found that the patients who had undergone breast biopsy were found to have anxiety due to pain, lack of knowledge, uncertainty regarding the biopsy results and fear of cancer. This caused anxiety levels among the participants in the control group to rise before the core needle biopsy and their anxiety remained high on the day of follow-up.

Similarly, Liao et al. ${ }^{15}$ found that informational and psychological supportive care post-operatively included educational pamphlets about breast cancer diagnosis and treatment, emotional support and follow-up telephone consultations. In Liao et al. ${ }^{15}$ the study group was found to have statistically significant lower mean anxiety scores than the control group before the core needle biopsy ( $p=0.017)$ and when receiving the diagnosis results $(p=0.001)$. In addition, Tan et al. ${ }^{26}$, who studied an educational program for women with newly diagnosed breast cancer, found the patients had higher knowledge and lower anxiety levels ( $p<0.001)$. This program provided handbooks on treatment guidelines, breast care 
nurse counselling, and educational videos on breast cancer.

The women that received the core needle biopsy after the telehealth program were seen to have statistically significant lower anxiety levels than before the telehealth program $(p<0.05)$. In this study, the participants in the study group were found to have the highest mean anxiety score before the telehealth program. These anxieties decreased after the program, both before the core needle biopsy and on the day of follow-up. The participants in the study group that were assigned to participate in the telehealth program received knowledge about the core needle biopsy via verbal teaching and handbooks. The patients were able to review the handbooks at home. Educating patients from the day of treatment at the hospital provided them with knowledge, understanding and the ability to prepare for events. This corresponded with the findings of Tantulawat and Patumwan ${ }^{27}$, who found that giving recommendations to the patients before receiving the core needle biopsy reduced their lack of understanding by 93 per cent.

In this study, the telehealth program provided channels for patients to communicate and make inquiries via the LINE app on smartphones, while they stayed at home. This supported the provision of complete information and psychological support by enabling nurses to speak with the patients, ask about their feelings and provide encouragment. These findings were consistent with the findings of Miller et al. ${ }^{8}$, who studied the effects of good communication between patients and radiotherapists on the day of a core needle biopsy procedure. The information included types, time and steps of methods of the procedure. In addition, the researcher spoke with the patients in order to prepare them for follow-up results, causing them to have lower anxiety $(p<0.001)$.
Obadina et al. ${ }^{18}$, who studied the effects of educational online support, used online education related to breast care, and answers from breast specialists to patients' questions. According to the findings from Obadina et al. ${ }^{18}$, the group participating in the online program at the core needle biopsy stage was found to have lower anxiety levels ( $p$ $=0.017$ ).

The participants in the present study were monitored for symptoms after the core needle biopsy and after discharge by the researcher sending messages to individual participants regarding potential symptoms after the procedure. Examples of these symptoms include abnormal bleeding, pain or discomfort. Most of the participants were found to have no abnormal symptoms while staying at home. Only 5.7 per cent of the participants (two participants) sent images to consult the researcher regarding wound characteristics and bruising of the breasts. The researcher consulted with the medical team regarding how to reply to the patients' inquiries. In contemporary studies, which used telehealth technology to monitor patients after breast cancer surgery, 75 per cent of the samples were found to have used the telehealth program. In those studies, many of the patients were concerned with the post-operative recovery time and other patients' experiences. One-third of the sample reported that the symptoms encountered while staying at home consisted of anxiety and pain. Fifteen per cent of the sample wanted to consult with a physician regarding the management of symptoms caused by anxiety and other side-effects ${ }^{28}$. In the area of mobile app development for breast cancer treatments of patients treated with chemotherapy, the patients were found to better perceive self-efficacy, symptom management and quality of life $(p<0.05)^{29}$.

\section{Conclusion}

The telehealth program in this study assessed only patients' anxiety levels. Current studies have reflected the effectiveness of the telehealth programs in assessing patients' quality of life and development to help them achieve better health outcomes. In this study, the participants that received the telehealth program were assessed for anxiety levels at each stage of the core needle biopsy and were monitored via long-range media continually until the day of follow-up to prepare them for every possible event, such as receiving bad biopsy results. Therefore, such a telehealth program can be used to care for patients receiving core needle biopsy so as to lower their anxiety levels.

\section{Implications for perioperative nursing}

The perioperative nurse should use the telehealth program to communicate with patients who have received a core needle biopsy. This telehealth program will be implemented in clinics by the multidisciplinary team including attending nurses and physicians. The nurse works as the efficiency coordinator who is aware of problems and the needs of the patient, in particular providing knowledge, health consultation and monitoring in the perioperative phase to educate patients about their upcoming surgery and recovery.

\section{Recommendations}

According to the findings, patients who participated in the telehealth program have lower anxiety. Therefore, the findings can be used to support the following recommendations for developing and implementing care of patients:

1. The telehealth program should be developed by using applications as another channel of 
communication with patients and should be used to monitor and care for other groups of patients undergoing biopsy.

2. Care policies should be developed that use technology to monitor patients' symptoms from when patients were at the hospital to when patients are home. In addition, the number of coordinators with special knowledge and expertise on the care of patients should be increased.

3. More qualitative studies should be conducted into the aetiology of anxiety among patients involved in this study to determine corrective guidelines and create care models for this group of patients in the future.

\section{Acknowledgements and disclosure}

The authors would like to acknowledge and thank Emily Schwager for proofreading the manuscript. The study was partially supported by Graduate Studies of Mahidol University Alumni Association. The authors declare no other financial conflicts of interest in this study.

\section{References}

1. Siegel RL, Miller KD, Jemal $A$. Cancer statistics, 2016. CA Cancer J Clin 2016;66(1):730.

2. Virani S, Bilheem S, Chansaard W, Chitapanarux I, Daoprasert K, Khuanchana S et al. National and subnational populationbased incidence of cancer in Thailand: Assessing cancers with the highest burdens. Cancers 2017;9(8):108.

3. Imsamran W, Chaiwerawattana A, Wiangnon S, Pongnikorn D, Suwanrungruang K, Sangrajrang $S$ et al. Cancer in Thailand: Vol. VIII, 2010-2012. Bangkok: National Cancer Institute; 2015.

4. Alsanabani JA, Gilan W, Saadi A. Incidence data for breast cancer among Yemeni female patients with palpable breast lumps. Asian Pac J Cancer Prev 2015;16(1):191-194.
5. Al-Rikabi A, Husain S. Increasing prevalence of breast cancer among Saudi patients attending a University referral hospital: A retrospective epidemiologic study. Croat Med J 2012;53(3):239.

6. Mazari F, Sharma N, Reid D, Horgan K. The need for triple assessment and predictors for diagnosis of breast cancer in patients < 40 years of age. Clin Radiol 2018;73(8):758. e19-758.e25.

7. Thanyarak Breast Center, University Hospital. Summary report of Thanyarak Breast Center. Bangkok: University Hospital; 2018 (in Thai).

8. Miller LS, Shelby RA, Balmadrid MH, Yoon S, Baker JA, Wildermann L et al. Patient anxiety before and immediately after imagingguided breast biopsy procedures: Impact of radiologist-patient communication. J Am Coll Radiol 2013;10(6):423-431.

9. Demir F, Donmez YC, Ozsaker E, Diramali A. Patients' lived experiences of excisional breast biopsy: A phenomenological study. J Clin Nurs 2008;17(6):744-751.

10. Lou Z, Li Y, Yang Y, Wang L, Yang J. Affects of anxiety and depression on health-related quality of life among patients with benign breast lumps diagnosed via ultrasonography in China. Int J Environ Res Public Health 2015;12(9):10587-10601.

11. Montgomery M, McCrone SH. Psychological distress associated with the diagnostic phase for suspected breast cancer: Systematic review. J Adv Nurs 2010;66(11):2372-2390.

12. Boinon D, Dauchy S, Charles C, Fasse L, Cano A, Balleyguier $C$ et al. Patient satisfaction with a rapid diagnosis of suspicious breast lesions: Association with distress and anxiety. Breast J 2018;24(2):154-160.

13. Deane KA, Gegner LF. Information needs, uncertainty, and anxiety in women who had a breast biopsy with benign outcome. Cancer Nurs 1998;21(2):117-126.

14. van Osch M, Sep M, van Vliet LM, van Dulmen S, Bensing JM. Reducing patients' anxiety and uncertainty, and improving recall in bad news consultations. J Health Psychol 2014;33(11):1382.

15. Liao MN, Chen PL, Chen MF, Chen SC. Effect of supportive care on the anxiety of women with suspected breast cancer. J Adv Nurs 2010;66(1):49-59.

16. Nandra K, Koenig G, DelMastro A, Mishler EA, Hollander JE, Yeo CJ. Telehealth provides a comprehensive approach to the surgical patient. Am J Surg. 2019;218(3):476-479.

17. van der Meij E, Anema JR, Otten RH, Huirne JA, Schaafsma FG. The effect of perioperative e-health interventions on the postoperative course: A systematic review of randomised and non-randomised controlled trials. PLoS One 2016;11(7):e0158612.
18. Obadina ET, Dubenske LL, McDowell HE, Atwood AK, Mayer DK, Woods RW et al. Online support: Impact on anxiety in women who experience an abnormal screening mammogram. Breast J 2014;23(6):743-748.

19. Panoinon S, Asdornwised U, Pinyopasakul W, O-Charoenrat P. The effectiveness of a day surgery discharge planning program on anxiety and satisfaction of care among women undergoing breast mass excision. J Nurs Sci (Supplement) 2010;28(4):28-36 (in Thai).

20. Chaithongsakul A. Using practice guideline when giving preoperative information: Effects on anxiety among patients with breast cancer prior to surgery. South Col J Nurs Public Health 2015;2(2):29-40 (in Thai)

21. Naylor MD, Shaid EC, Carpenter D, Gass B, Levine C, Li J et al. Components of comprehensive and effective transitional care. J Am Geriatr Soc 2017;65(6):1119-1125.

22. Soo MS, Jarosz JA, Wren AA, Soo AE, Mowery YM, Johnson KS et al. Imaging-guided coreneedle breast biopsy: Impact of meditation and music interventions on patient anxiety, pain and fatigue. J Am Osteopath Coll Radiol 2016;13(5):526-534.

23. Gaberson KB. The effect of humorous distraction on preoperative anxiety. AORN J 1991;54(6):1258-1261.

24. Brocken P, Prins JB, Dekhuijzen PR, van der Heijden HF. The faster the better?-A systematic review on distress in the diagnostic phase of suspected cancer, and the influence of rapid diagnostic pathways. Psychooncology 2012;21(1):1-10.

25. Harding MM. Incidence of distress and associated factors in women undergoing breast diagnostic evaluation. West J Nurs Res 2014;36(4):475-494.

26. Tan ML, Lee KH, Yong WS, Rodgers C. The effects of a video-based education in women with newly diagnosed breast cancer in Singapore. Support Care Cancer 2018:1-7.

27. Tantulawat M, Patumwan P. The results of patient counselling using video clips during core needle biopsy in female patients revealed breast lumps. Chiangrai Med J 2018;10(1):141-148 (in Thai).

28. Noble N, Mackenzie L, Carey M, Proietto A, Sanson-Fisher R, Walker $\mathrm{G}$ et al. Crosssectional survey to inform the development of a telehealth support model: A feasibility study for women undergoing breast cancer surgery. Pilot Feasibility Study 2019;5(1):46.

29. Zhu J, Ebert L, Liu X, Wei D, Chan SW-C. Mobile breast cancer e-support program for Chinese women with breast cancer undergoing chemotherapy (part 2): Multicenter randomized controlled trial. JMIR Mhealth Uhealth 2018;6(4):e104. 